\title{
Investigation of an Equation with Logarithmic Coupling with Momentum in Dirac Equation
}

\author{
Mash-Huda Rahman Shipra*, Syed Badiuzzaman Faruque \\ Department of Physics, Shahjalal University of Science and Technology, Sylhet, Bangladesh \\ Email address: \\ smashhuda@gmail.com (Mash-Huda R. S.) \\ ${ }^{*}$ Corresponding author
}

To cite this article:

Mash-Huda Rahman Shipra, Syed Badiuzzaman Faruque. Investigation of an Equation with Logarithmic Coupling with Momentum in Dirac Equation. International Journal of High Energy Physics. Vol. 7, No. 2, 2020, pp. 32-36. doi: 10.11648/j.ijhep.20200702.11

Received: July 7, 2020; Accepted: July 27, 2020; Published: August 10, 2020

\begin{abstract}
The free particle solutions of the relativistic Dirac equation are characterized by plane waves with infinite uncertainty in position. However, many practical implementations of the solutions require a wave packet configuration, which can be utilized to represent a localized Dirac particle. Unlike the traditional wave packet generation method by superposing multiple plane waves, this study of ours presents an alternative approach towards obtaining a wave packet solution of a free particle relativistic Dirac equation. In this paper, we present Dirac's free particle equation with a modification in the generalized momentum. The modification is achieved by coupling the momentum with a spatially varying logarithmic function, and this alteration does not affect the relativistic dispersion relation of the particle. Moreover, a solution of this modified Dirac equation is provided as well, which has been calculated using a trial wave function. The wave function solution is carried out in one dimension, where it behaves as a wave packet for a given ratio of the envelope parameter to the reduced Planck's constant greater than unity, where the envelope parameter regulates the width of the wave packet. The solution, being subject to this constraint, represents a bound particle with spin and a continuous energy spectrum.
\end{abstract}

Keywords: Modified Dirac Equation, Localized Wave Packet, Generalized Momentum, Logarithmic Coupling

\section{Introduction}

A particle that is described by its phase space coordinates classically, can be analogously represented by a state vector in the quantum realm. That associated state vector would be localized around that particle's phase space position. The evolution of the localized state vector would mimic the evolution of the particle in space and time.

Working with a particle in a quantum mechanical framework is challenging since the localization of that particle is limited by Heisenberg's uncertainty principle.

In order to formulate a quantum state which would resemble a classical particle, it is very effective to make use of wave packets with finite widths. The wave packet treatment is particularly effective in practical implementations [1-5]. Numerous attempts to generate a wave packet solution on both relativistic and nonrelativistic regimes have been made over the years using various techniques [6-8]. The wave packet investigation of the relativistic Dirac equation is particularly important because it serves a major function in nanophysics
[9-12].

The conventional method of constructing a wave packet is by linearly superposing multiple plane waves. As an alternative approach, some have endeavored to construct a single state wave packet on account of the nonlinearity imposed on a Rydberg electron by a linearly polarized electromagnetic field $[13,14]$ in a nonrelativistic arrangement. Furthermore, in the relativistic arena, an analogous investigation has been successfully conducted using the free particle Dirac wave equation [15] which uses coupling of a spatially varying potential with the generalized momentum.

It is feasible to produce wave packet solutions of the free particle Dirac equation by performing a coupling with the generalized momentum in the Dirac Hamiltonian. The modification in the momentum does the job of packing the otherwise sinusoidal wave into a wave packet with finite width in position [15].

In this paper, we have presented a solution in the form of a wave packet for a Dirac wave equation with appropriate modification of the momentum operator. The modification has 
been made by coupling the generalized momentum of the free particle Dirac Hamiltonian with a spatially varying logarithmic function. This modified Dirac equation, along with its properties, has been presented within the subsequent section followed by its solution in $(1+1)$ freedom.

\section{Dirac Equation with a Modified Generalized Momentum}

The modified Dirac equation contains in its generalized momentum operator a spatially varying logarithmic function and for definite energy $E$ thus takes the following form:

$$
\left[c \vec{\alpha} \cdot(\vec{p}-i q \ln \vec{r})+\beta m c^{2}\right] \psi(\vec{r})=E \psi(\vec{r})
$$

Here,

and

$$
\vec{\alpha}=\left(\begin{array}{ll}
0 & \vec{\sigma} \\
\vec{\sigma} & 0
\end{array}\right)
$$

$$
\beta=\left(\begin{array}{cc}
I & 0 \\
0 & -I
\end{array}\right)
$$

are Dirac $4 \times 4$ matrices, $c$ is the speed of light in vacuum, $\vec{p}$ and $\vec{r}$ are the momentum and position of the Dirac particle respectively and $q$ serves as the envelope parameter which regulates the shape of the wave packet. The wave function $\psi(\vec{r})$ is a stationary energy eigenstate.

The modified Dirac equation in the one-dimensional case in which the motion of a particle can be considered along z-direction with momentum $p_{z}$ appears as

$$
\left[c \alpha_{z}\left(p_{z}-i q \ln z\right)+\beta m c^{2}\right] \psi(z)=E \psi(z)
$$

The wave function $\psi(z)$ can be considered as a four-component column vector

$$
\psi(z)=\left(\begin{array}{l}
u_{1} \\
u_{2} \\
u_{3} \\
u_{4}
\end{array}\right)
$$

These four components operated by (2) produce the following four equations

$$
\begin{aligned}
c\left(p_{z}-i q \ln z\right) u_{3} & =\left(E-m c^{2}\right) u_{1} \\
c\left(p_{z}-i q \ln z\right) u_{1} & =\left(E+m c^{2}\right) u_{3} \\
-c\left(p_{z}-i q \ln z\right) u_{4} & =\left(E-m c^{2}\right) u_{2} \\
-c\left(p_{z}-i q \ln z\right) u_{2} & =\left(E+m c^{2}\right) u_{4}
\end{aligned}
$$

Now, (4) and (5) are coupled together, as are (6) and (7). It is convenient to derive equations for two of the component variables independent of the other two. Therefore, by taking $u_{2}=u_{4}=0$, the following two equations can be obtained

$$
\begin{aligned}
& \left(p_{z}-i q \ln z\right)\left(p_{z}-i q \ln z\right) u_{1}=\left(\frac{E^{2}}{c^{2}}-m^{2} c^{2}\right) u_{1} \\
& \left(p_{z}-i q \ln z\right)\left(p_{z}-i q \ln z\right) u_{3}=\left(\frac{E^{2}}{c^{2}}-m^{2} c^{2}\right) u_{3}
\end{aligned}
$$

Similarly, for $u_{1}=u_{3}=0$ two more equations are obtained for $u_{2}$ and $u_{4}$ similar to (8) and (9).

Hence the four equations satisfied by the four components of the spinor in (3) are all identical. Taking into consideration the identicality of the four equations, it is reasonable to find the solution for only one equation, namely the one governing $u_{1}$ which will apply to all of the four equations.

Substituting the momentum operator

$$
p_{z} \rightarrow-\mathrm{i} \hbar \frac{\partial}{\partial \mathrm{z}}
$$

gives the following partial differential equation,

$$
\frac{\hbar}{q} z \frac{\partial^{2} u_{1}}{\partial z^{2}}+2 z \ln z \frac{\partial u_{1}}{\partial z}+\frac{q}{\hbar} z \ln ^{2} z u_{1}+K^{\prime} u_{1}=0
$$

where,

$$
K^{\prime}=\frac{1}{q \hbar}\left(\frac{E^{2}}{c^{2}}-m^{2} c^{2}\right)+1
$$

\section{Solution}

The method of solving the equation (10) involves choosing a suitable trial wave function solution to compose a localized wave packet containing an unknown spatially dependent function $\phi(z)$. Later, an equation governing the unknown $\phi(z)$ is derived on condition that the trial wave function satisfy (10). Finally, putting the solution derived from that equation into the trial wave function gives the solution of the modified Dirac equation.

A choice of trial wave function solution for the aforementioned equation can be considered in the form

$$
u_{1}(z)=\frac{\hbar}{q} \phi(z) \exp \left[-\frac{q}{\hbar} \int \ln z d z\right]
$$

The reasons for this choice are twofold.

Firstly, the integral term within the exponential function brings forth a logarithmic term when differentiated with respect to $z$, while the minus sign in front assists it in simplifying the equation by eliminating identical terms.

Secondly, the $\hbar / q$ multiple coupled with the $q / \hbar$ term within the exponential serves the purpose of reducing the equation down to its simplest form for $\phi(z)$ by omitting those terms therein.

The trial wave function in (11) acted on by (10) yields the following governing equation for $\phi(z)$,

$$
z \frac{\partial^{2} \phi}{\partial z^{2}}+K \phi=0
$$

where,

$$
K=\frac{E^{2}}{\hbar^{2} c^{2}}-\frac{m^{2} c^{2}}{\hbar^{2}}
$$

is the square of the wave number $k$.

Equation (12) can be reduced to the Bessel equation of order 1 [16] which consequently produces

$$
\phi(z)=\mathrm{A} \sqrt{z} J_{1}(2 \sqrt{K z})+\mathrm{B} \sqrt{z} Y_{1}(2 \sqrt{K z})
$$


The function is a linear combination of both the first and second kind Bessel functions of order one, where $A$ and $B$ are arbitrary constants, $J_{1}$ and $Y_{1}$ are the Bessel functions of the first and second kind respectively, both of order 1 .

\section{Result}

The solution to the aforementioned Dirac equation is, therefore,

$$
u_{1}(z)=\frac{\hbar}{q}\left[\mathrm{~A} \sqrt{z} J_{1}(2 \sqrt{K z})+\mathrm{B} \sqrt{z} Y_{1}(2 \sqrt{K z})\right] \exp \left[-\frac{q}{\hbar} \int \ln z d z\right]
$$

The Dirac spinor in (3) assumes two independent forms for the two cases of spin up and down:

$$
\begin{gathered}
\Psi_{u p}(z)=\mathrm{N}\left(\begin{array}{l}
1 \\
0 \\
1 \\
0
\end{array}\right) \frac{\hbar}{q}\left[\mathrm{~A} \sqrt{z} J_{1}(2 \sqrt{K z})+\mathrm{B} \sqrt{z} Y_{1}(2 \sqrt{K z})\right] \exp \left[-\frac{q}{\hbar} \int \ln z d z\right] \\
\Psi_{\text {down }}(z)=\mathrm{N}\left(\begin{array}{l}
0 \\
1 \\
0 \\
1
\end{array}\right) \frac{\hbar}{q}\left[\mathrm{~A} \sqrt{z} J_{1}(2 \sqrt{K z})+\mathrm{B} \sqrt{z} Y_{1}(2 \sqrt{K z})\right] \exp \left[-\frac{q}{\hbar} \int \ln z d z\right]
\end{gathered}
$$

Equation (16) satisfies the case when $u_{2}=u_{4}=0$ and (17) satisfies when $u_{1}=u_{3}=0 . N$ is the normalization factor in both equations.

The normalization factor $N$ can be determined by

$$
\int \psi^{*} \psi d z=1
$$

The solutions (16) and (17) represent a wave packet localized in position and is also an energy eigenstate with definite energy eigenvalue,

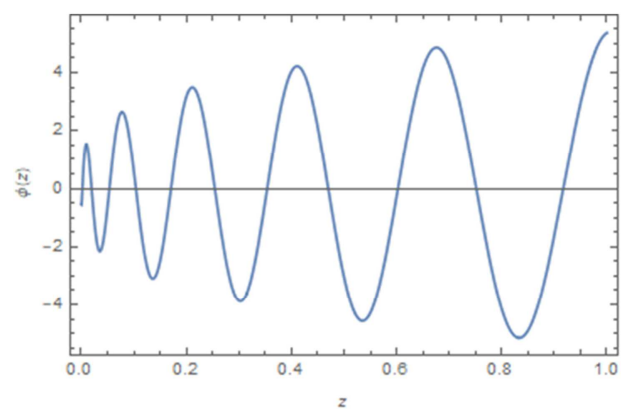

(a)

Figure 1. $\phi(z)$ vs $z$ graph for $A=2, B=3$ (a) $K=300$ and (b) $K=3000$.

The wave function itself, on the other hand, exhibits the desired wave packet form Figures 2-4 as long as the envelope parameter is greater than the reduced Planck's constant $\hbar$. However, Figure 5 shows that it tends to diverge for an

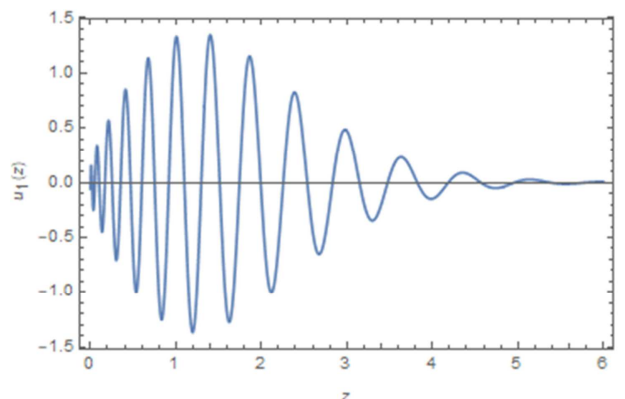

(a)

$$
E= \pm \sqrt{K \hbar^{2} c^{2}+m^{2} c^{4}}= \pm \sqrt{k^{2} \hbar^{2} c^{2}+m^{2} c^{2}}
$$

where wave number $k=\sqrt{K}$ which is evidently independent of the envelope parameter $q$. Hence, it is evident that the dispersion relation persists despite the modification in the Hamiltonian. The eigenvalue is a continuous energy spectrum with both positive and negative values.

In the graphs of the general Bessel function solution $\phi(z)$ as shown in Figure 1, the envelope of the plane wave can be seen to diverge over the positive $\mathrm{z}$-direction. The values of the constants A, B and $\mathrm{K}$ are chosen arbitrarily.

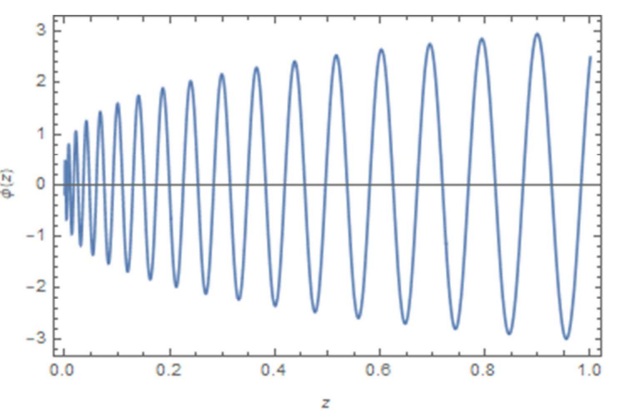

(b)

envelope parameter smaller than $\hbar$. The graphs in figures 2-5 have been plotted for the unnormalized top component of (16). The values of the envelope parameter $q$ have been chosen as multiples of the reduced Planck's constant $\hbar$.

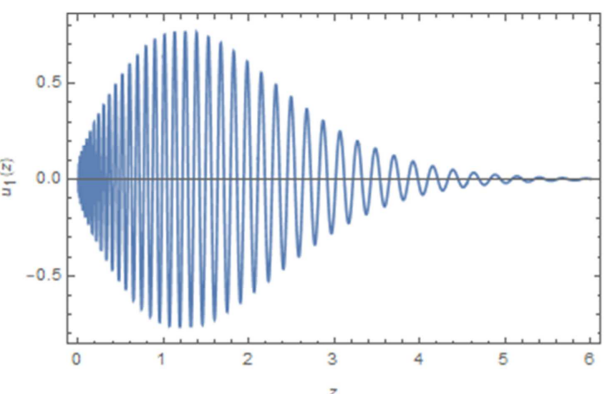

(b)

Figure 2. $u_{1}(z)$ vs z graph for $A=2, B=3$ where $q=\hbar$ (a) $K=300$ and (b) $K=3000$ 


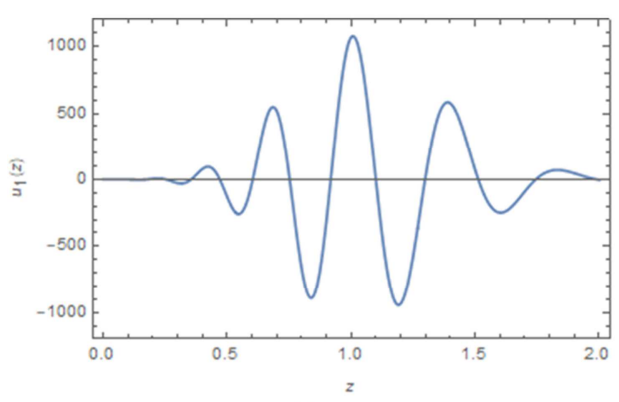

(a)

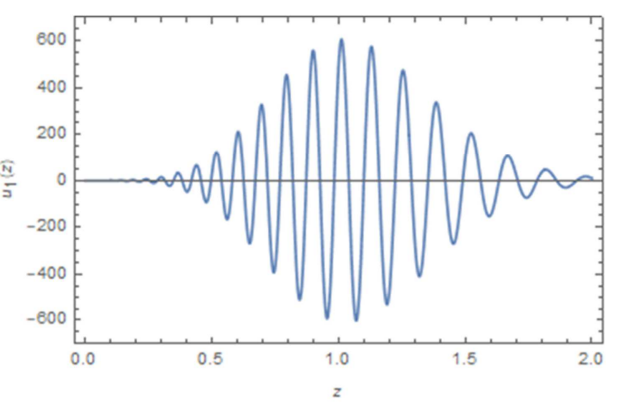

(b)

Figure 3. $u_{1}(z)$ vs z graph for $A=2, B=3$ where $q=10 \hbar$ (a) $K=300$ and (b) $K=3000$.

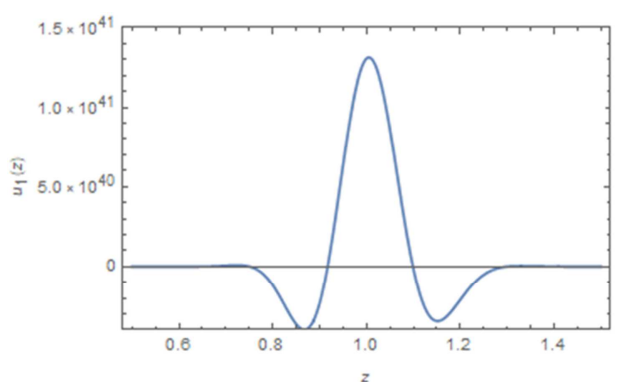

(a)

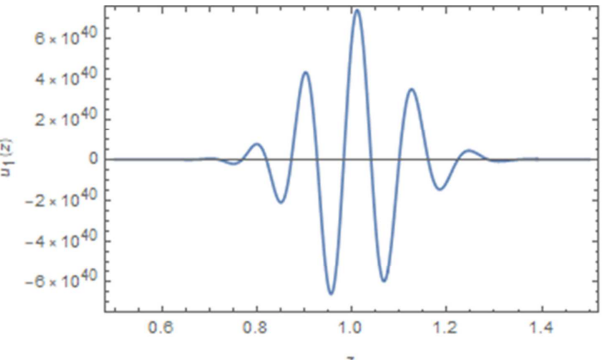

(b)

Figure 4. $u_{1}(z)$ vs z graph for $A=2, B=3$ where $q=100 \hbar$ (a) $K=300$ and (b) $K=3000$

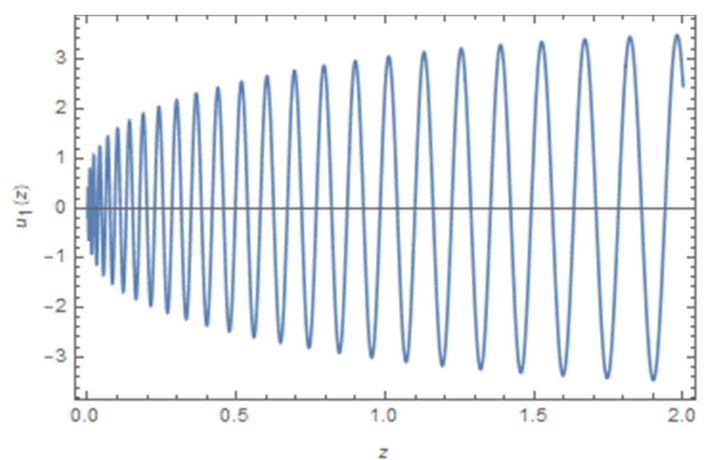

Figure 5. $u_{1}(z)$ vs z graph for $A=2, B=3$ where $q=0.1 \hbar$ and $K=3000$.

\section{Discussion}

The foregoing investigation of the free particle Dirac equation modified by introducing a logarithmic term into the generalized momentum has yielded some intriguing results. Firstly, the function $\phi(z)$, which is a linear combination of Bessel functions of the first and second kind of order one, diverges infinitely as $z$ increases Figure 1. Yet, the complete wave function is successfully enveloped into a wave packet.

Secondly, the envelope parameter q acts as a deciding factor between a localized wave packet and diverging wave train for the solution depending on its value compared to the reduced Planck's constant. For q greater than $\hbar$, the solutions generated in Figures 2-4 are wave packets localized in position whose width is regulated by the envelope parameter itself.

Thirdly, the increase of $q$ invokes a tremendous increase in the amplitude of the envelope of the wave packets at the same time, a reduction in their width. However, the envelope amplitude decreases with increasing squared wave number $K$. The envelope parameter $q$, as well as the constant $K$, affects the wavenumber. An increase in $q$ results in a decrease in wavenumbers. The graphs Figures 2-4 also suggest that the wave packets show a dispersive tendency as distance increases.

Even though the squared wavenumber $K$ is independent of the envelope parameter, as can be seen from (13), the wave packets in the figures contain fewer wave numbers as $q$ increases. This phenomenon occurs due to the fact that the width of the wave packets becomes smaller with larger values of $q$ and, therefore, can accommodate lesser numbers of waves.

Finally, as the envelope parameter assumes a value less than the reduced Planck's constant, the solution turns into an indefinitely diverging plane wave mimicking somewhat the structure of the $\phi(z)$ vs $z$ curve from Figure 1 .

\section{Conclusion}

In this paper, we presented an alternative to the conventional wave packet generation technique by multiple plane wave superposition. We instead used a spatially varying logarithmic coupling with the generalized momentum in the free particle Dirac Hamiltonian and successfully demonstrated that wave packing is possible through a slight modification to the free particle Dirac equation. The logarithmic term $-i q \ln \vec{r}$ introduced into the generalized momentum does the job of wave packing depending on the value of the envelope parameter q.

The study is conducted on a $(1+1)$ freedom considering the particle's motion in the $\mathrm{z}$-direction. The solutions are definite energy eigenstates with definite spins $(1 / 2$ or $-1 / 2)$ as well. The energy eigenvalue of the Dirac equation has a continuous spectrum.

The modification in the generalized momentum packs 
continuous wave trains into a wave packet with a finite width in position depending on the $q / \hbar$ ratio. If the ratio takes on a value less than unity, the wave function diverges indefinitely rather than being enveloped into a packet.

To conclude, we have presented in this article an unconventional method for producing a localized wave function from the otherwise unlocalized infinite wave trains utilizing the wave packing characteristic of the equation itself unlocked through simple modification.

\section{Acknowledgements}

The author Mash-Huda Rahman Shipra is immensely indebted to her supervisor Prof. Syed Badiuzzaman Faruque.

\section{References}

[1] Garraway, B. M., \& Suominen, K. A. (1995). Wave-packet dynamics: new physics and chemistry in femto-time. Reports on Progress in Physics, 58 (4), 365.

[2] Herek, J. L., Materny, A., \& Zewail, A. H. (1994). Femtosecond control of an elementary unimolecular reaction from the transition-state region. Chemical physics letters, 228 (1-3), 15-25.

[3] Julienne, P. S., Smith, A. M., \& Burnett, K. (1992). Theory of collisions between laser cooled atoms. In Advances in atomic, molecular, and optical physics (Vol. 30, pp. 141-198). Academic Press.

[4] Leo, K., Shah, J., Göbel, E. O., Damen, T. C., Schmitt-Rink, S., Schäfer, W., \& Köhler, K. (1991). Coherent oscillations of a wave packet in a semiconductor double-quantum-well structure. Physical review letters, 66 (2), 201.

[5] Ruprecht, P. A., Holland, M. J., \& Burnett, K. (1994). Dynamical effects in atom optics. Physical Review A, 49 (6), 4726.
[6] Bialynicki-Birula, I., \& Bialynicka-Birula, Z. (2017). Relativistic electron wave packets carrying angular momentum. Physical review letters, 118 (11), 114801.

[7] Park, S. T. (2012). Propagation of a relativistic electron wave packet in the Dirac equation. Physical Review A, 86 (6), 062105 .

[8] Strange, P. (2017). Angular momentum of a relativistic wave packet. Physical Review A, 96 (5), 052103.

[9] Szabó, L. Z., Benedict, M. G., Czirják, A., \& Földi, P. (2013). Relativistic electron transport through an oscillating barrier: Wave-packet generation and Fano-type resonances. Physical Review B, 88 (7), 075438.

[10] Demikhovskii, V. Y., Maksimova, G. M., Perov, A. A., \& Frolova, E. V. (2010). Space-time evolution of Dirac wave packets. Physical Review A, 82 (5), 052115.

[11] Demikhovskii, V. Y., Maksimova, G. M., \& Frolova, E. V. (2008). Wave packet dynamics in a two-dimensional electron gas with spin orbit coupling: Splitting and zitterbewegung. Physical Review B, 78 (11), 115401.

[12] Rusin, T. M., \& Zawadzki, W. (2008). Zitterbewegung of electrons in graphene in a magnetic field. Physical Review B, 78 (12), 125419.

[13] Delande, D., Zakrzewski, J., \& Buchleitner, A. (1995). A wave packet can be a stationary state. EPL (Europhysics Letters), 32 (2), 107.

[14] Buchleitner, A., \& Delande, D. (1995). Nondispersive electronic wave packets in multiphoton processes. Physical review letters, 75 (8), 1487.

[15] Faruque, S. B., Shuvo, S. D., Moniruzzaman, M., \& Das, P. K. (2019). Localized states of modified Dirac equation. Sust J. Sci. Tech. 29 (1).

[16] Bell, W. W. (2004). Special functions for scientists and engineers. Courier Corporation. 